doi:10.1017/\$1041610219000711

\section{Effect of a dual-task exercise to motor and memory function for Japanese older individuals in depopulated rural districts: preliminary intervention research from 2016 to 2019}

In Japan, the first country to experience a super-aging society, the development of independent rehabilitative care for older individuals in depopulated rural areas is an indispensable challenge to decrease a high incidence of weak psychosomatic state to need care. Interestingly, some studies provide evidence that dual-task training has been shown to induce improvements in targeted motor and cognitive function (Suzuki et al., 2013; Tait et al., 2017). However, these findings remain for older individuals leaving in relatively urban neighborhoods. This preliminary study examined the effect of dual-task training for Japanese older persons in depopulated rural areas.

This research was conducted from 2016 to 2019, including the Katagami's study, and our previous studies at Oga and Higashinaruse areas (Kume et al., 2017, 2019). Thirty-five participants (age [mean $\pm S D] 72.0 \pm 5.4$ years; education, $11.4 \pm 2.3$ years; \%female, $94 \%$ ) without a history of neurological, psychiatric disorders, and dementia were recruited from rural areas in Akita prefecture. The participants performed a 90 -minute exercise program comprised of stretching, muscle strength training, a step-up/ down aerobic exercise using the stepstool, and dual-task training (e.g. the step-up/down movement while working naming tasks, a colorful ladder exercise combined the Stroop task) for once per one or two weeks for six months (Suzuki et al., 2013). Physical and cognitive measurements were performed at baseline and after the six-month intervention. The physical measurement consisted of the Timed Up and Go test (TUG), grip strength (GS), and the Sit to Stand-5 (SS-5). The National Center for Geriatrics and Gerontology Functional Assessment Tool (Shimada et al., 2017) was also applied to examine the effect to cognitive function before and after the intervention, comprised of the Word Recognition Memory (WRM) and the tablet version of the Trail Making Test - part A and part B (TMT-A, TMT-B). This study was approved by the Ethics Committee of Akita University (No. 1769).

The result of the Wilcoxon signed-rank test indicated that a required time (second) of the SS- 5 was significantly shorter (median [interquartile range], pretest $=6.7[1.8]$, post-test $=5.4[1.6], \mathrm{p}<0.0001)$, and the WRM score was significantly improved (pre $=12.0$ [5.0], post $=13.0$ [4.6], $p=0.008$ ) after the intervention. However, a significant difference between preand post-tests was not observed in other physical and cognitive domains, such as the TUG $(p=0.44)$, the GS $(p=0.63)$, the TMT-A $(p=0.83)$ and TMT-B $(\mathrm{p}=0.72)$.

Our preliminary findings lead us to believe that a dual-task exercise program can be potentially effective to the enhancement of motor and memory function for Japanese older persons in depopulated rural districts, in accordance with an improvement of memory scores following dual-task training (Suzuki et al., 2013; Tait et al., 2017). Whether these results generalize to enhancements for subjects in other areas remains unclear but warrants examination involving a randomized controlled trial.

\section{Conflict of Interest}

None.

\section{Description of authors' roles}

Authors contributed to data collections, data interpretation and writing, and revision of the report.

\section{Acknowledgements}

This work for the center of community (COC) project was supported by funding of the Japanese Ministry of Education, Culture, Sports, Science and Technology.

\section{References}

Kume, Y., et al. (2017). Pilot study: the application of a multicomponent exercise for older people in a depopulated rural area. Geriatrics and Gerontology International, 17, 2639-2640.

Kume, Y., et al. (2019). Effective strategy of the multicomponent exercise program for older individuals in a depopulated rural region. International fournal of Gerontology, 13, 153-154.

Shimada, H., Makizako, H., Park, H., Doi, T. and Lee, S. (2017). Validity of the National Center for Geriatrics and Gerontology-Functional Assessment Tool and Mini-Mental State Examination for detecting the incidence of dementia in older Japanese adults. Geriatrics Gerontology International, 17, 2383-2388. 
Suzuki, T., et al. (2013). A randomized controlled trial of multicomponent exercise in older adults with mild cognitive impairment. PLoS One, 8, e61483.

Tait, J. L., Duckham, R. L., Milte, C. M., Main, L. C. and Daly, R. M. (2017). Influence of sequential vs. simultaneous dual-task exercise training on cognitive function in older adults. Frontiers in Aging Neuroscience, 9, 368.

Yu Kume, ${ }^{1}$ Tomoe Fujita, ${ }^{2}$ Sachiko Uemura, ${ }^{3}$ Shoko Inomata, ${ }^{2}$ Megumi Tsugaruya, ${ }^{1}$ Akiko Sato, ${ }^{4}$ Yoriko Nakamura, ${ }^{2}$ Yuki ITAKURA ${ }^{5}$ and HideTAKa OTA ${ }^{5}$

${ }^{1}$ Department of Occupational Therapy, Graduate School of Medicine, Doctorial Course in Health Sciences, Akita University, Akita, Japan

${ }^{2}$ Department of Nursing for Community Living, Graduate School of Medicine, Doctorial Course in Health Sciences, Akita University, Akita, Japan
${ }^{3}$ Department of Physical Therapy, Graduate School of Medicine, Doctorial Course in Health Sciences, Akita University, Akita, Japan

${ }^{4}$ Graduate School of Medicine, Center for Aging in Place, Akita University, Akita, Japan

${ }^{5}$ Advanced Research Center for Geriatric Medicine, Akita University, Akita, Japan

Correspondence should be addressed to: Yu Kume, Department of Occupational Therapy, Graduate School of Medicine, Doctorial Course in Health Sciences, Akita University, 1-1-1 Hondo, Akita, 010-8543, Japan. Phone: +81-18-884-6556;

Fax: +81-18-884-6556.

Email: kume.yuu@hs.akita-u.ac.jp 